九州大学学術情報リポジトリ

Kyushu University Institutional Repository

\title{
Profile-Decomposing Output of Multi-Channel Odor Sensor Array
}

Zheng, Xiaofan

Kyushu University

Tomiura, Yoichi

Kyushu University

Hayashi, Kenshi

Kyushu University

Soeda, Takaaki

Kyushu University

ht tp://hdl. hand le. net/2324/4479593

出版情報: ECS Meeting Abstracts. MA2020-01，pp.1855-，2020-05-10. IOP Publishing バージョン：

権利関係 : 


\section{Introduction}

This paper proposes a method for profile-decomposing the output from a segmented multisubpixels 2-D sensor array, that is, a method for obtaining the spatial concentration distribution of each component odor using the output from the image sensor.

Image sensors corresponding to our sense of vision and the advanced image analysis technology have greatly changed our social life. Similarly, the development of an odor image sensor capable of measuring odor characteristics in space and the method for profile-decomposing the output from the sensor is expected to bring a great change to our social life.

Two of the co-authors of this paper developed an odor image sensor device to measure residual odor traces on the surface. In the device, many chips composing of 4 different odor sensors that response differently to odors (we call this chip as a segmented 4-subpixels sensor) are arranged in 2-dimmensions. The schematic is shown in Figure1. This odor image sensor can be used to measure four-dimensional odor characteristics at each point.

Non-negative Matrix Factorization is a method of decomposing a non-negative matrix into a non-negative weight matrix and a non-negative feature matrix. When the number of component odors, that is, the number of basis vectors is given, the output data from device can be decomposed to a concentration matrix expressing concentration of each component odor at each point and a profile matrix expressing response pattern of segmented 4-subpixels sensor to each component odor with Nonnegative Matrix Factorization (NMF).

We assume the followings:

(1) A linear relationship holds true between the concentration of component odor and the output of each segmented subpixel sensor.

(2) All segmented $C$-subpixels sensor chips arranged in 2 dimensions have the same response property to odors.

(3) The concentration of component odor in the segmented $C$-subpixels sensor chip is uniform.

If these three assumptions hold true, the output data of device can be completely expressed as the product of the concentration matrix and the profile matrix. However, in reality these assumptions do not always hold and we consider these assumptions to be approximately true by miniaturization of the chip and calibration of the sensor output. As for determining the number of component odors, in this study, we verified how effective the four information criteria are for estimating the number of component odors in profile-decomposition by simulation experiments using artificial data including noise with various variance. In addition, profile-decomposition and four kinds of 
information criteria are performed on the data when using the device to measure the mixture of two component odors.

\section{Applying NMF and model selection}

We assume that there are $I$ chips on the device, and $C$ sensors on each chip, there are $K$ component odors on the area covered by $I$ chips. Let the element $a_{i k}$ in the matrix A (concentration matrix) denote the concentration of the $k$-th component odor at the position $i$, and the element $b_{k c}$ in matrix $\mathrm{B}$ (profile matrix) represent the output of the $c$-th odor sensor when measuring unit concentration of $k$-th component odor. According to the three assumptions mentioned in the introduction, the output of the device represented by matrix X can be expressed as (1), here E is a noise matrix that follows a normal distribution.

In practical applications, on the contrary, the output matrix is decomposed into the concentration matrix and the profile matrix. In this paper, we use (2) as the objective function. The algorithm proposed by Daniel D. Lee and H. Sebastian Seung is shown as Figure2. An example of decomposing artificial output data without noise is shown in Figure3.

The number of component odors is the number of basis vectors in NMF. In this study, we conducted four kinds of information criterion to estimate the number of component odors. Equation (3), (4), (5), (6) are the formulas for calculating the four information criteria which are AIC, AICc, BIC and MDL. Here $k$ is the degree of freedom of the model, N is the number of elements in the X, $\mathrm{L}$ is the maximum likelihood function.

\section{Experiment}

In the simulation experiment, we randomly generate matrices A and B. The values of the elements in matrix A range from 0 to 1 , and the values of the elements in matrix B range from 0 to 25 . According to (1), the elements in the matrix $\mathrm{X}$ is calculated as (7), $e_{i j}$ is generated from normal distribution $N\left(0, \sigma^{2}\right)$. Here if $x_{i j}<0, x_{i j}=0$. We used each of four information criteria to estimate the number of component odors with different $\sigma^{2}$. We conducted the estimation experiments 360 times for one value of $\sigma^{2}$. The results are shown in Figure4.

For the real data, we decomposed the data obtained by the odor image sensor device and estimated the number of component odors. The actual number of component odors was two. The results are shown in Table1. Among the three methods, only AICc correctly estimated the number of component odors.

\section{Conclusion}


We propose the method based on NMF and information criterion for analyzing the output data obtained by the odor image sensor device. We used four kinds of information criterion to estimate the number of component odors. For the actual observed data by the odor image sensor device, AICc correctly estimated the number of component odors. 